\title{
POSTER
}

\section{Lésions du nerf alvéolaire inférieur secondaires aux traitements endodontiques : Mise au point et suivi de 2 cas cliniques}

\section{Castro $\mathrm{R}^{1,2}$, Lan $\mathrm{R}^{1}$, Catherine $\mathrm{J}-\mathrm{H}^{1,2,3}$, Guyot $\mathrm{L}^{2,3}$}

1 - Service de chirurgie orale, Pavillon odontologie, CHU Timone, Marseille

2 - Service de chirurgie maxillo-faciale, chirurgie orale et chirurgie plastique, Hôpital Nord, AP-HM, Université AixMarseille, Marseille

3 - UMR 7268 ADES Aix-Marseille Université/EFS/CNRS, Marseille

romain.castro@ap-hm.fr

Mots clés : Lésions endodontiques / Nerf alvéolaire inférieur / Paresthésie labio-mentonnière

La proximité anatomique entre les racines des prémolaires et molaires mandibulaires avec le canal mandibulaire explique la survenue des lésions du nerf alvéolaire inférieur pouvant être associées à des thérapeutiques endodontiques.

Trop souvent négligée par les praticiens, une extrusion de matériau dans le canal mandibulaire peut entraîner des séquelles neurologiques allant des paresthésies à des anesthésies dans le territoire des afférences du nerf alvéolaire inférieur. Le contrôle radiologique en fin de traitement endodontique par les chirurgiens-dentistes doit donc être systématique afin de mettre en évidence, le plus tôt possible, tout dépassement de pâte endodontique au delà de l'apex.

II s'agit, au travers d'une revue de la littérature s'étendant sur les 15 dernières années et l'exposition de 2 cas cliniques, de décrire les principaux mécanismes et la physiopathologie de lésions nerveuses, les symptômes dont se plaint les patients couramment et la prise en charge médico-chirurgicale.

Le premier cas est une patiente ayant bénéficier d'une résection apicale de curetage du matériau au sein du canal mandibulaire droit 3 mois après le traitement endodontique iatrogène. Nous la recevons en consultation 2 ans après le traumatisme nerveux et la prise en charge médicale au pôle antidouleur d'Avignon qui en a découlé s'est avéré efficace avec un traitement à base de prégabaline.

Le deuxième cas est une patiente qui a été opéré 3 semaines après le traitement endodontique avec dépassement de matériau au sein du canal mandibulaire droit. Le curetage n'a pu être complet en première intention et la patiente est revu à 9 mois afin d'établir un traitement par prégabaline au vue d'importantes paresthésies persistantes. Un mois après le début du traitement, la patiente décrit une amélioration de ses symptômes.

La prise en charge chirurgicale précoce d'un dépassement de matériau est de bon pronostic, en revanche un retard diagnostique ou un délai trop important entre le traumatisme nerveux et la consultation au sein d'une équipe spécialisée s'associent souvent à des lésions irréversibles. 
Un délai de 15 jours est le maximum quant à la décision d'un geste chirurgical d'ablation du matériel. Au delà, un traitement médical symptomatique, le plus souvent à base de Prégabaline, est conduit. Un acte chirurgical après ce délai est parfois indiqué dans certains cas particuliers notamment quand un processus infectieux y est associé.

Enfin, un arbre décisionnel thérapeutique est proposé afin de guider les praticiens face à ces situations.

\section{Références}

Olsen JJ et al. J Cranio-Maxillofac Surg. sept 2014;42(6):757-62.

López-López J et al. Int Endod J. 1 janv 2012;45(1):98-104.

Pogrel MA et al. J Am Dent Assoc. janv 2007;138(1):65-9. 